\title{
A rational approach to optimize feed profiles for the maximization of productivity of secreted proteins expressed in Pichia pastoris Michael Maurer ${ }^{1}$, Brigitte Gasser ${ }^{1}$, Manfred Kühleitner ${ }^{2}$ and Diethard Mattanovich*1,3
}

Address: ${ }^{1}$ University of Natural Resources and Applied Life Sciences Vienna, Department of Biotechnology, Institute of Applied Microbiology, Vienna, Austria, ${ }^{2}$ University of Natural Resources and Applied Life Sciences Vienna, Department of Integrative Biology, Institute of Mathematics, Vienna, Austria and ${ }^{3}$ School of Bioengineering, University of Applied Sciences FH-Campus Vienna, Austria

* Corresponding author

from The 4th Recombinant Protein Production Meeting: a comparative view on host physiology

Barcelona, Spain. 2I-23 September 2006

Published: 10 October 2006

Microbial Cell Factories 2006, 5(Suppl I):SI4 doi:I0.II86/I475-2859-5-SI-SI4

(C) 2006 Maurer et al; licensee BioMed Central Ltd.

\section{Background}

Yeast processes for the production of secreted recombinant proteins are usually performed in fed batch. Although it is established that the productivity of such processes is defined by the space time yield, there is a lack of knowledge correlating physiological data to process related data defined by the feed profile. Typically these fed-batches employ either exponential or linear feed rates. Based on our experience, both protocols are not optimal solutions for secreted proteins.

Using a Pichia pastoris clone expressing the Fab fragment of the human monoclonal antibody 2F5 under the glyceraldehyde phosphate dehydrogenase (GAP) promoter, we determined the correlation of specific product formation rates $\left(\mathrm{q}_{\mathrm{P}}\right)$ as well as biomass yields $\left(\mathrm{Y}_{\mathrm{X} / \mathrm{S}}\right)$ in relation to a wide range of specific growth rates $(\mu)$. Measuring transcript levels of the product specific genes as well as 49 host genes related to growth, protein synthesis, oxygen and nutrient limitation responses, and protein secretion stress (UPR, ERAD, posttranslational processing), enabled the determination of net specific transcription rates. These parameters were determined in chemostat cultures in a range of dilution rates $(=\mu)$ between 0.02 and $0.2 \mathrm{~h}^{-1}$.

\section{Results}

$\mathrm{q}_{\mathrm{P}}$ showed an asymptotic relation to $\mu$, which could be modeled by a Monod-type equation. $\mathrm{Y}_{\mathrm{X} / \mathrm{S}}$ could be described by approximation of the maintenance coeffi- cient $\mathrm{m}$ and the maximum yield coefficient. The specific transcription rates of the product related genes (under control of the GAP promoter), as well as those of glycolytic (e.g. PFK1) and ribosomal genes correlated with $\mu$. TCA cycle related genes, and some stress genes were more independent of growth, while UPR controlled genes were more strongly expressed at high $\mu$.

The determination of a relation of $\mathrm{q}_{\mathrm{P}}, \mathrm{Y}_{\mathrm{X} / \mathrm{S}}$ and $\mu$ enabled the development of an optimization model for fed batch based on the Microsoft Excel Solver. This optimization tool allows easily to impose different constraints on the model, like minimum product concentration or maximum biomass concentration, both being key parameters for downstream processing. Depending on the constraints, different combinations of exponentially and linearly increasing feed rates were calculated as optimal feed protocols. Model predictions were compared with actual fed batch data.

\section{Conclusion}

These data provide evidence that recombinant protein secretion is decoupled from growth and specific mRNA synthesis at higher specific growth rates, thus approaching a saturation level at higher $\mu$. Consequently the development of $\mu$ over time is a critical parameter for an optimal fed batch process. 